\title{
Enhanced radiation characteristics for antipodal Vivaldi antenna using high permittivity dielectric director
}

\author{
Rabiaa Herzi*, Moufida Bouslama and Ali Gharsallah \\ Laboratory of Electronics, Department of Physics \\ Faculty of Science of Tunis, 2092 El Manar, Tunisia \\ *rabiaaherzi@yahoo.fr
}

Received 3 April 2017; Revised 10 May 2017; Accepted 18 May 2017; Published 22 June 2017

\begin{abstract}
In this paper, we investigate the influence of higher permittivity dielectric director on the radiation performances of an antipodal Vivaldi antenna. An elliptical dielectric director with high permittivity is inserted in an antipodal Vivaldi antenna aperture in order to ameliorate the radiation characteristics of the antenna. Due to the capacity of elevated permittivity dielectric to confine and guide energy in the desired direction, an increment of $4 \mathrm{~dB}$ in the gain of the antenna is obtained. This antenna, which covers an ultra-wide frequency band of $146.8 \%$ from $2.3 \mathrm{GHz}$ to $15 \mathrm{GHz}$, has approximately regular radiation patterns with reduced side lobe level and narrower beamwidth. In the interest to achieve radar application necessities, the proposed antenna is exploited to develop an antenna array which consists of four connected elements. Adding dielectric directors can significantly enhance the radiations characteristics of the antenna and reduce the mutual coupling inter-elements. So using four elements with dielectric director in the antenna array can achieve the same results obtained with eight elements without directors. This can decrease the used number of elements that form the antenna array.
\end{abstract}

Keywords: Dielectric director; high permittivity; antipodal Vivaldi antenna (AVA); ultra-wideband (UWB); antenna array.

\section{Introduction}

Recently, compact antenna array with almost constant radiation patterns over an ultra-wideband of frequency has attracted many researches. ${ }^{1}$ These antennas are required in many applications such as radar sensors, high-performance imaging systems and satellite communications. ${ }^{2}$

Antipodal Vivaldi antenna, which is a traveling wave antenna, was first presented by Gazit. ${ }^{3}$ It has an ultra large band of frequency and acceptable directivity, but suffers from beam tilting, elevated side lobe level (SLL) and variable radiation patterns over the operating band, particularly at the low frequencies. ${ }^{3,4}$ These characteristics seem to be insufficient for target applications.

Several methods have been suggested to enhance the radiation performances of the Vivaldi antenna such as loading slots on each side of the antenna. ${ }^{4,5}$ Inserting a dielectric director with higher permittivity is a good solution to ensure better radiation performances. ${ }^{6}$ It can extensively improve the directivity of the antenna. ${ }^{7}$

Due to its capability to confine and guide the electrical and magnetic fields, the dielectric director with high permittivity can conduct the radiation of the antenna in the desired direction and reduce reflections from the extremities of the antenna, which can ameliorate the gain, narrow the beamwidth of the principal lobe and inhibit the beam tilting of the antenna. ${ }^{8}$ In addition, many dielectrics with high permittivity have the capacity to operate over a wide band of frequency ( $2 \mathrm{GHz}$ to $40 \mathrm{GHz}$ ). ${ }^{9}, 10$
Moreover, the usual method to improve the radiation of the antenna and to achieve the radar requirements is to develop an antenna array. This leads to the establishing of a grand size antenna system with uncondensed configuration. ${ }^{1,11}$ Besides, placing a dielectric with high permittivity in the front of each element that structures the antenna array can considerably reduce the used number of elements and reduce the mutual coupling inter-antennas. This approach offers a compact structure with a small size of the antenna array system. ${ }^{6,12}$

In this paper, an antipodal Vivaldi antenna (AVA) with elliptical high permittivity dielectric director and four connected elements antenna are proposed. The powerful influence of the high permittivity dielectric on the radiation performances of the antenna is studied.

In Sec. 2, the single antenna with dielectric director (AVA-D) and the effects of the high dielectric constant of the director are detailed. Section 3 presents the antenna array design and discusses the obtained results, in order to investigate the capacity of high permittivity dielectrics to improve the radiation performances of the antenna array. Finally, Sec. 4 sums up this paper.

\section{Single Antenna with High Permittivity Dielectric Director}

In this section, the single antenna structure and the effects of the added dielectric rod with high permittivity are analyzed

This is an Open Access article published by World Scientific Publishing Company. It is distributed under the terms of the Creative Commons Attribution 4.0 (CC-BY) License. Further distribution of this work is permitted, provided the original work is properly cited. 
and given. The antenna is designed and simulated using the CST Microwave Studio.

Figure 1 illustrates the structure of the proposed antenna. The geometry of the simple AVA is shown in Fig. 1(a), which consists of top and bottom exponential copper layers printed on an RF35 substrate with 3.5 of permittivity and 0.76 of thickness. The exponential taper profile follows fundamental equations defined as

$$
\begin{gathered}
Y= \pm\left(A e^{R x}+B\right), \\
A=\frac{y_{2}-y_{1}}{e^{R x_{2}}-e^{R x_{1}}}, \\
B=\frac{y_{1} e^{R x_{2}}-y_{2} e^{R x_{1}}}{e^{R x_{2}}-e^{R x_{1}}} .
\end{gathered}
$$

$\left(x_{1}, y_{1}\right),\left(x_{2}, y_{2}\right)$ are the peak and bottom points, respectively, of the exponentially tapered curve and $R$ is the exponential factor.

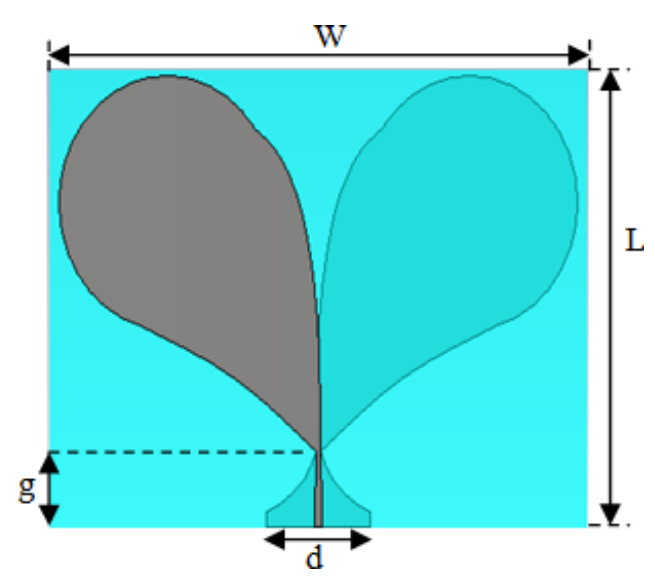

(a)

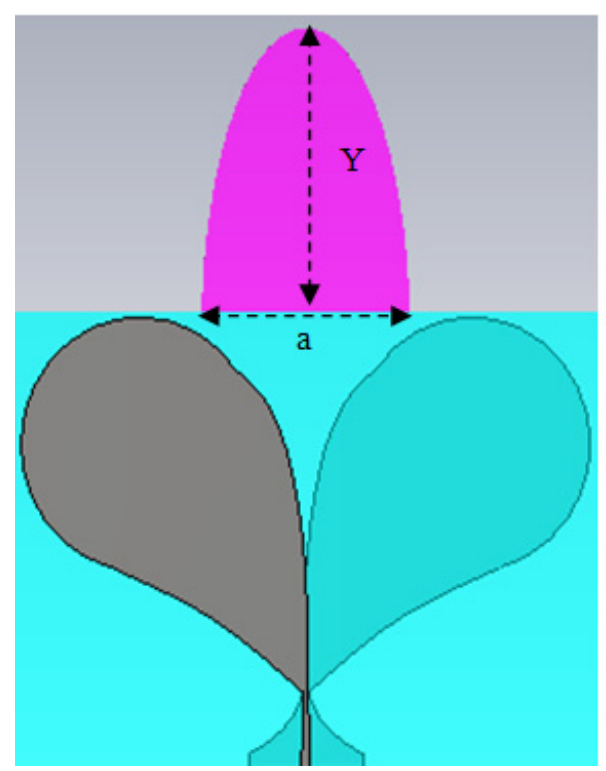

(b)

Fig. 1. AVA without (a) and with (b) elliptical dielectric director.
Table 1. Dimensions of the design antenna (mm).

\begin{tabular}{cccccc}
\hline W & g & L & d & Y & a \\
\hline 89.4 & 15 & 86 & 24 & 38 & 24 \\
\hline
\end{tabular}

The dimensions of the antenna are listed in Table 1.

Figure 1(b) shows AVA with the elliptical dielectric director, which has the same thickness of the antenna substrate. It is placed in the front of the antenna aperture in order to guide the radiation of the antenna in its direction. The substrate of the director is the Rogers R03010 with 10.2 of permittivity and $24 \mathrm{~mm}$ of width. Its length $(Y)$ is determined using the Eq. (4).

$$
Y=\frac{\lambda_{S}}{\sqrt{\varepsilon}_{D}}
$$

where $\lambda_{S}$ is the wavelength that corresponds to the low-frequency cut of the antenna and $\varepsilon_{D}$ is the dielectric constant of the director.

The size of the director can be reduced by the augmenting of the permittivity. Hence, in order to find a small structure, the director permittivity is chosen to be higher. The designed length of the director is about $38 \mathrm{~mm}$.

The radiation factor of the dielectric director can be defined by the following relation:

$$
Q_{\mathrm{RAD}}=\varepsilon_{D}^{3 / 2} .
$$

This can demonstrate that the radiation factor is augmented by the increasing of the permittivity of the dielectric director. Indeed, the electrical and magnetic fields will be more concentrated around the director by increasing its permittivity.

As a result, the reflection coefficients of the AVA and the AVA with the dielectric director, which are calculated by the formula (5), are shown in Fig. 2.

$$
\begin{gathered}
S_{11}=\frac{Z_{\text {in }}-Z_{c}}{Z_{\text {in }}+Z_{c}}, \\
S_{11}(\mathrm{~dB})=10 \underset{10}{\log }\left|\left(\frac{Z_{\text {in }}-Z_{c}}{Z_{\text {in }}+Z_{c}}\right)\right| .
\end{gathered}
$$

It can be seen that both antennas present good input match over a wide frequency range $(2.3-15 \mathrm{GHz})$, where the reflection coefficients are less than $-10 \mathrm{~dB}$. The antennas exhibit a wide bandwidth of $146.8 \%$ that is calculated using the following relation:

$$
\mathrm{BR} \%=\frac{f_{\max }-f_{\min }}{f_{c}} \times 100,
$$

where $f_{\max }, f_{\min }$ and $f_{c}$ are, respectively, the high cut frequency, the low cut frequency and the frequency center.

Consequently, the insertion of the director with high permittivity in the antenna did not influence the reflection coefficient. 


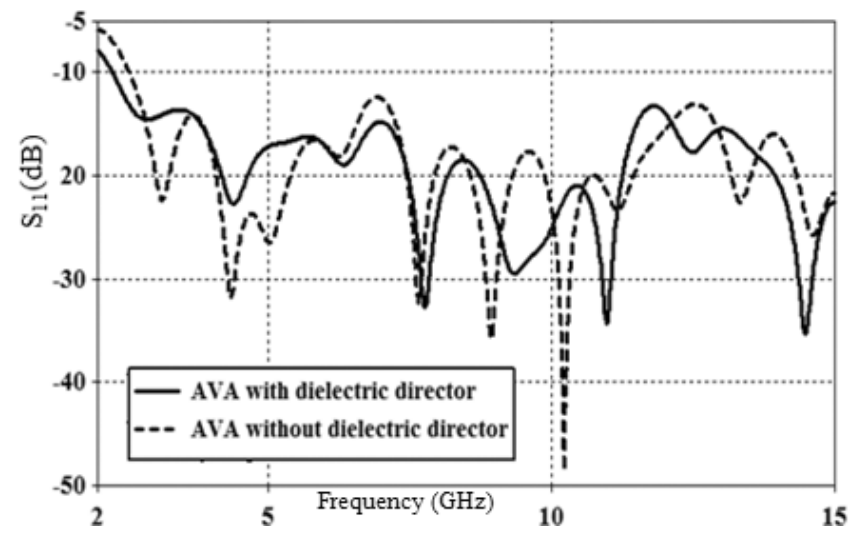

Fig. 2. Reflection coefficients of the conventional antenna and the AVA with director.

In order to investigate the effect of the high permittivity dielectric director, the radiation patterns in the $E$-plane and $H$-plane of the antenna without director and with director with different permittivities are compared in Fig. 3. It is clear that the adding of the dielectric director has significantly improved the antenna radiation characteristics. Moreover, the best results are attained by using a director with 10.2 of permittivity. Table 2 depicts a comparison between the



(a)

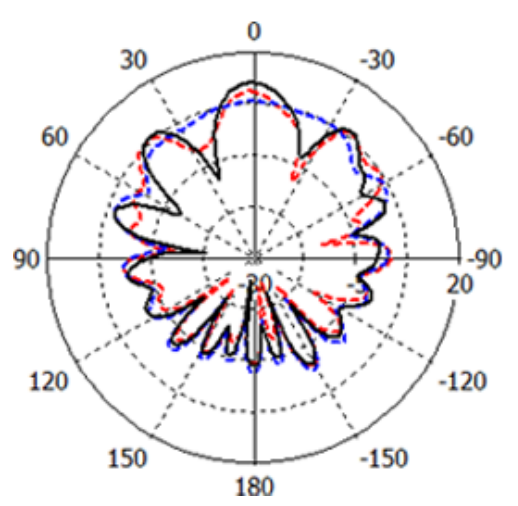

(b)

Fig. 3. Radiation patterns of the AVA with and without dielectric director at $10 \mathrm{GHz}$ in $E$-plane (a) and $H$-plane (b).
Table 2. Radiation characteristics of both antennas at $7.5 \mathrm{GHz}$

\begin{tabular}{lcccccccc}
\hline & \multicolumn{3}{c}{$E$-plane } & & \multicolumn{3}{c}{$H$-plane } \\
\cline { 2 - 4 } Type of antenna & $\begin{array}{c}\text { Gain } \\
(\mathrm{dB})\end{array}$ & $\begin{array}{c}\text { SLL } \\
(\mathrm{dB})\end{array}$ & $\begin{array}{c}\text { HPBW } \\
\left({ }^{\circ}\right)\end{array}$ & & $\begin{array}{c}\text { Gain } \\
(\mathrm{dB})\end{array}$ & $\begin{array}{r}\text { SLL } \\
(\mathrm{dB})\end{array}$ & $\begin{array}{c}\text { HPBW } \\
\left({ }^{\circ}\right)\end{array}$ \\
\hline AVA & 7.93 & -9.2 & 51 & & 7.9 & 1.9 & 90.1 \\
AVA-D $\left(\varepsilon_{r}=6\right)$ & 10.2 & -10.2 & 34 & & 10.3 & -2.1 & 19.6 \\
AVA-D $\left(\varepsilon_{r}=10.2\right)$ & 12.4 & -10.9 & 32 & & 12.5 & -3.8 & 19.3 \\
\hline
\end{tabular}

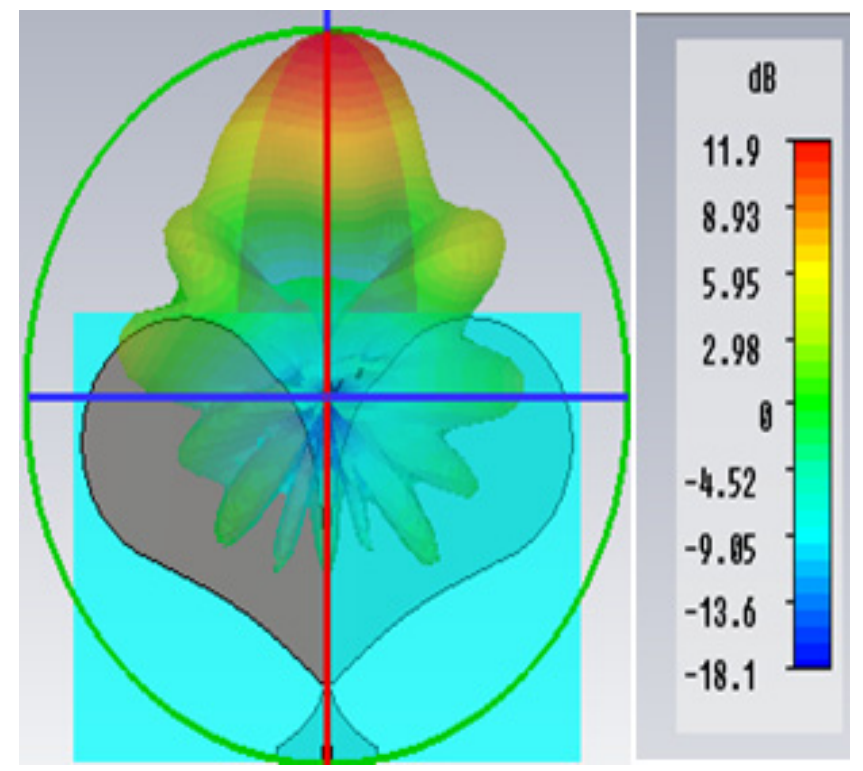

Fig. 4. Radiation pattern in 3D of the proposed antenna at $7.5 \mathrm{GHz}$.

radiation performances of different antennas for $E$-plane and $H$-plane. It is indicating that the directivity of the antenna has increased by increasing the permittivity of the director. In addition, by using the director with higher dielectric constant, the half-power beamwidth (HPBW) gets extensively narrower and the SLL is decreased. Consequently, increasing of the permittivity of the director can notably ameliorate the radiation performances of the antenna.

Figure 4 shows the 3D radiation pattern of the antenna with dielectric Director with permittivity of 10.2 at $7.5 \mathrm{GHz}$. It can be seen that the energy is concentrated and guided in the direction of the director. This can demonstrate the effect of the high permittivity director in guiding the waves to its summit.

\section{AVA Array with Elliptical Dielectric Directors}

The antenna array structure is developed to achieve the radiation performances required in many applications such as radar sensing and satellite communications.

In our implementation, the antenna array design consists of four connected AVA elements. Four elliptical dielectric 


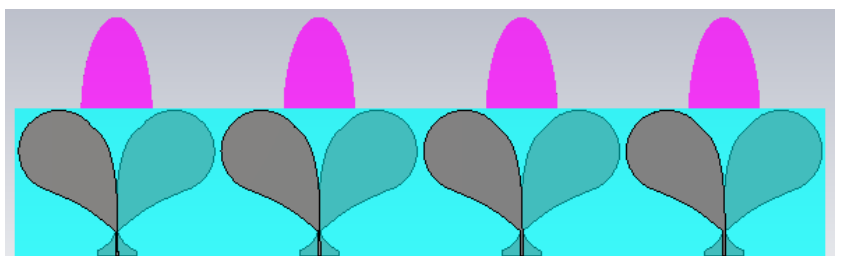

Fig. 5. Four elements array.

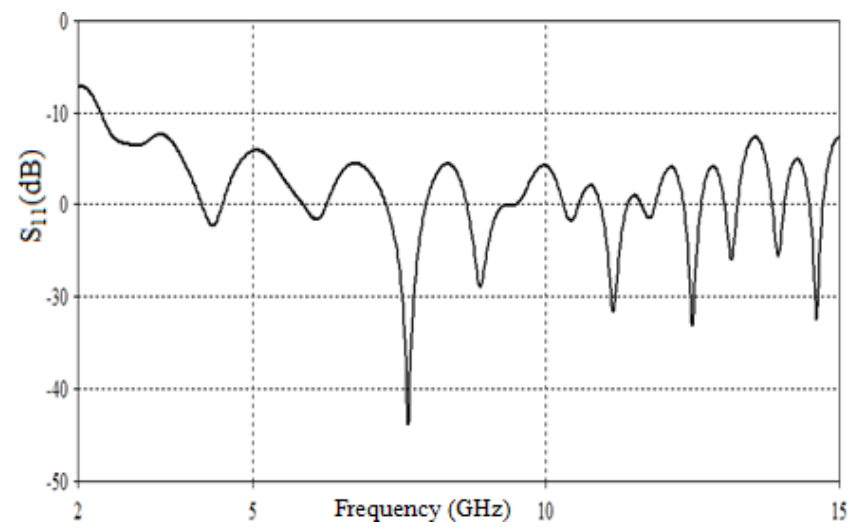

Fig. 6. Reflection coefficient of the antenna array.

Directors with 10.2 of permittivity are inserted in the front of the antennas apertures, shown in Fig. 5.

Figure 6 presents the reflection coefficient of the proposed antenna array. It can be seen that the input return loss is less than $(-10 \mathrm{~dB})$ over the band of $2.3 \mathrm{GHz}$ to $15 \mathrm{GHz}$. It is practically similar to the reflection coefficient of the single antenna.

On the other hand, reducing the mutual coupling interelements of the antenna array, which can damage the radiation performances of the array, is a principal goal in the antenna array design. It can be reduced by increasing the spacing between the elements; nevertheless, the size of the antenna array will be enlarged. However, the important

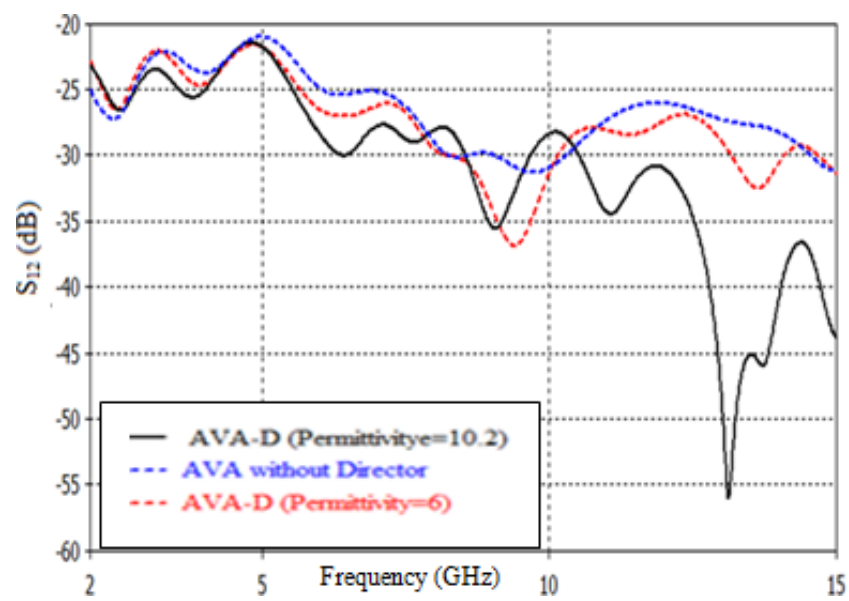

Fig. 7. Mutual coupling between the adjacent elements in the antenna array.



(a)



(b)

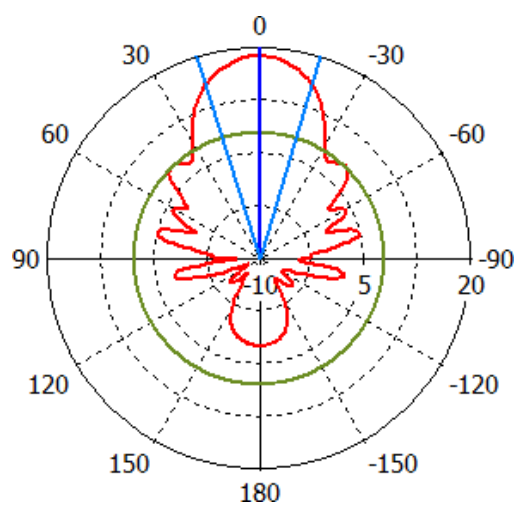

(c)

Fig. 8. Radiation patterns of the AVA array with higher permittivity directors at (a) $4 \mathrm{GHz}$, (b) $7.5 \mathrm{GHz}$ and (c) $10 \mathrm{GHz}$.

advantage of inserting the directors with high permittivity is its capability to reduce the mutual coupling between the interelements. Figure 7 presents the transmission coefficient between the elements in the simple antenna array, antenna array with directors with 6 and with 10.2 of permittivity. It is indicated that the mutual coupling of the antenna array with the higher permittivity directors is better than that of the other arrays mainly at the high frequencies, due to its capability to reduce the dispersion and to confine and guide the electrical field in its direction. 
Table 3. Radiation characteristics of the antenna array configuration.

\begin{tabular}{lcccc}
\hline & Gain $(\mathrm{dB})$ & SLL $(\mathrm{dB})$ & HPBW $\left({ }^{\circ}\right)$ & Beam tilting $\left({ }^{\circ}\right)$ \\
\hline $4 \mathrm{GHz}$ & 14 & -9.3 & 81 & 1 \\
$7.5 \mathrm{GHz}$ & 17.7 & -12.4 & 43 & 0 \\
$10 \mathrm{GHz}$ & 18.7 & -10.7 & 34.2 & 0 \\
\hline
\end{tabular}

The radiation patterns of the antenna array with directors with 10.2 of permittivity at different frequencies are shown in Fig. 8. It can be seen that the patterns are almost similar over the operating band. In addition, improved gain with narrow HPBW is attained at the different frequency. This proposed structure can avoid the beam tilting of the simple antenna array where the main lobe pointed at $0^{\circ}$. Table 3 recapitulates the radiation performances of the proposed antenna array at different frequencies.

To conclude, as a result of the capacity of the high permittivity director to conduct the energy in its summit and to reduce the losses of the energy, a directive antenna system with small size, narrower beamwidth and with almost constant radiation patterns over an ultra-wide frequency band is achieved.

\section{Conclusion}

In this paper, we have investigated the power of high permittivity dielectric director to improve the radiation performances of the antipodal Vivaldi antenna. The director has the ability to confine the fields and inhibit the reflections in the extremities of the antenna that results in a directive antenna with reduced SLL, narrow beam width and minimum beam tilting.

Additionally, in the antenna array structure, inserting the dielectric directors is more efficient in improving the radiation performances, reducing the mutual coupling between the elements and inhibiting the beam tilting. This method allows to obtain an array structure with minimum numbers of elements which is necessary to reduce the size of the antenna system. In conclusion, the proposed structure presents a good candidate for radar applications and satellite communications.

\section{References}

${ }^{1}$ R. Herzi et al., Antipodal Vivaldi Antenna Array reduced mutual coupling for UWB radars applications, 15th Inter. Conf. on Sciences \& Techniques of Automatic Control \& Computer Engineering (STA 2014).

${ }^{2}$ B. Allen, M. Dohler, E. E. Okon, W. Q. Malik, A. K. Brown and D. J. Edwards, Ultra Wideband Antennas and Propagation for Communications, Radar and Imaging, Chapter 20 (Wiley, NJ, 2006), pp. 437-448.

${ }^{3}$ E. Gazit, Improved design of the Vivaldi antenna, Proc. Inst. Elect. Eng. - Microwave, Antennas, Propagation, Vol. 135(2), pp. 89-92, 1988.

${ }^{4}$ J. Bai et al., Modified compact antipodal Vivaldi antenna for 4-50-GHz UWB application, IEEE Trans. Microw. Theory Tech. 59(4) (2011).

${ }^{5}$ R. Herzi et al., A tuneable antipodal Vivaldi antenna for UWB applications, 10th Int. Multi-Conf. Systems, Signals and Devices (SSD-13), Hammamet, Tunisia, March 2013.

${ }^{6} \mathrm{R}$. Kazemi et al., Dielectric rod antenna array with substrate integrated waveguide planar feed network for wideband applications, IEEE Trans. Antennas Propag. 60(3) 1051 (2012).

${ }^{7}$ R. K. Chaudhary et al., Three-element multilayer multipermittivity cylindrical dielectric resonator antenna for wideband applications with omnidirectional radiation pattern and low crosspolarization, Microw. Opt. Technol. Lett. 54(9) (2012).

${ }^{8} \mathrm{~J}$. P. Pousi et al., High permittivity dielectric rod waveguide as an antenna array element for millimeter waves, IEEE Trans. Antennas Propag. Vol. 58, No. 3, pp. 714-719, March, 2010.

${ }^{9}$ Y. Geand and K. P. Esselle, A dielectric resonator antenna for UWB applications, IEEE Trans. Antennas Propag. (2009).

${ }^{10}$ W. M. Wu et al., Studies of permittivity and permeability of dielectric matrix with cuboid metallic inclusions in different orientations, J. Adv. Dielectr. 4(4) 1450032-1 (2014).

${ }^{11}$ Y. Yang et al., Development of an ultra wideband Vivaldi antenna array, Proc. IEEE Int. Symp. Antennas Propag. Soc. (2005) pp. 606-609.

${ }^{12} \mathrm{~J}$. Bourqui et al., Balanced antipodal Vivaldi antenna with dielectric director for near Fieeld microwave imaging, IEEE Trans. Antennas Propag. 58(7), 2318 (2010). 